\title{
The Label Splitting Problem
}

\author{
J. Carmona \\ Universitat Politècnica de Catalunya, Spain \\ jcarmona@lsi.upc.edu
}

\begin{abstract}
The theory of regions was introduced by Ehrenfeucht and Rozenberg in the early nineties to explain how to derive (synthesize) an event-based model from an automaton. To be applicable, the theory relies on stringent conditions on the input automaton. Although some relaxation on these restrictions has been done in the last decade, in general not every automaton can be synthesized while preserving its behavior. A crucial step for a non-synthesizable automaton is to transform it in order to satisfy the synthesis conditions. This paper revisits label splitting, a technique to satisfy the synthesis conditions through renaming of problematic labels. For the first time, the problem is formally characterized and its optimality addressed.
\end{abstract}

\section{Introduction}

The synthesis problem [4] consists in building a Petri net [8] that has a behavior equivalent to a given automaton (transition system). The problem was first addressed by Ehrenfeucht and Rozenberg [5] introducing regions to model the sets of states that characterize marked places in the Petri net. The theory is applicable to elementary transition systems, a proper subclass where additional conditions are required, and for which the synthesis produces a Petri net with isomorphic behavior. These restrictions were significantly relaxed in [3], introducing the subclass of excitation-closed transition systems, where not isomorphism but bisimilarity is guaranteed. The theory of this paper is within the subclass of excitation-closed transition systems.

When synthesis conditions do not hold, the Petri net derived might represent a proper superset of the initial behavior [1], and therefore any faithful use of such Petri net is rather limited. To overcome this problem, one might force the synthesis conditions by transforming the initial transition system. The work in [3] was the first in addressing this problem, introducing label splitting as a technique that can be applied when excitation-closure is not satisfied. The technique is based on relabeling the transitions of a particular event in the transition system with new copies of the same event, thus preserving the event name but considering each new copy as a new event with respect to the synthesis conditions. However, [3] only presented the technique as a heuristic to progress into excitation-closure.

The new copies produced by the label splitting technique increase the complexity of the Petri net derived: each new copy will be transformed into a transition, and hence the label splitting problem is to find an optimal sequence of 
splittings that induces the minimal number of transitions in the derived Petri net.

The label splitting technique presented in this paper is a particular one: it is defined on the sets of states computed when searching for regions in state-based synthesis methods $[1,3]$. These sets, called essential, are the building blocks used in this paper to decide the labels to split. The methods for label splitting in the aforementioned work also use the essential sets for label splitting, but as described previously, only in a heuristic manner.

In summary, this paper presents a novel view on the label splitting technique. First, we show how label splitting for excitation closure is nothing else than computing the optimal coloring of a graph, i.e., the chromatic number. Second, we characterize the conditions under which an optimal label splitting can be derived to accomplish excitation closure. Finally, we present an algorithm that can be used when excitation closure can not been attained by a single application of the label splitting technique presented in this paper. This algorithm is based on a relaxation of the label splitting problem that can be mapped into the weighted set cover problem.

For the sake of clarity, the theory of this paper will be presented for the class of safe (1-bounded) Petri nets. The contribution can be extended with no substantial change for the class of general ( $k$-bounded) Petri nets.

\section{Preliminaries}

\subsection{Finite transition systems and Petri nets}

Definition 1 (Transition system). A transition system is a tuple $\left(S, E, A, s_{i n}\right)$, where $S$ is a set of states, $E$ is an alphabet of actions, such that $S \cap E=\emptyset, A \subseteq S \times E \times S$ is a set of (labeled) transitions, and $s_{\text {in }}$ is the initial state.

We use $s \stackrel{e}{\rightarrow} s^{\prime}$ as a shortcut for $\left(s, e, s^{\prime}\right) \in A$, and we denote its transitive closure as $\stackrel{*}{\rightarrow}$. A state $s^{\prime}$ is said to be reachable from state $s$ if $s \stackrel{*}{\rightarrow} s^{\prime}$. Let $\mathrm{TS}=\left(S, E, A, s_{i n}\right)$ be a transition system. We consider connected transition systems that satisfy the following axioms: i) $S$ and $E$ are finite sets, ii) every event has an occurrence: $\forall e \in E: \exists\left(s, e, s^{\prime}\right) \in A$, and iii) every state is reachable from the initial state: $\forall s \in S: s_{\text {in }} \stackrel{*}{\rightarrow} s$.

Definition 2 (Petri net [8]). A Petri net is a tuple $\mathrm{PN}=\left(P, T, F, M_{0}\right)$ where $P$ and $T$ represent finite disjoint sets of places and transitions, respectively, and $F \subseteq(P \times T) \cup(T \times P)$ is the flow relation. The initial marking $M_{0} \subseteq P$ defines the initial state of the system.

For a node $n$ (place or transition) of a Petri net, $\bullet n(n \bullet)$ is the predecessor (successor) set of $n$ in $F$. A transition $t \in T$ is enabled in a marking $M$ iff $\bullet \subseteq M$. The firing of $t$ results in a new marking $M^{\prime}$, with one less token in $\bullet t$ and one more token in $t \bullet$. A marking $M^{\prime}$ is reachable from $M$ if there is a sequence of firings 
$\sigma=t_{1} t_{2} \ldots t_{n}$ that transforms $M$ into $M^{\prime}$, denoted by $M[\sigma\rangle M^{\prime}$. A sequence of transitions $\sigma=t_{1} t_{2} \ldots t_{n}$ is a feasible sequence if $M_{0}[\sigma\rangle M$, for some $M$. The set of all markings reachable from the initial marking $M_{0}$ is called its Reachability Set. The Reachability Graph of a Petri net PN (RG(PN)) is a transition system in which the set of states is the Reachability Set, the events are the transitions of the net and a transition $\left(M_{1}, t, M_{2}\right)$ exists if and only if $M_{1} \stackrel{t}{\rightarrow} M_{2}$.

\section{$2.2 \quad$ Regions and synthesis}

The theory of regions provides a way to go from transition systems to Petri nets. We now review this theory (the interested reader can refer to $[1,3-5,7]$ for a complete overview). Let $S^{\prime}$ be a subset of the states of a transition system, $S^{\prime} \subseteq S$. If $s \notin S^{\prime}$ and $s^{\prime} \in S^{\prime}$, then we say that transition $\left(s, a, s^{\prime}\right)$ enters $S^{\prime}$. If $s \in S^{\prime}$ and $s^{\prime} \notin S^{\prime}$, then transition $\left(s, a, s^{\prime}\right)$ exits $S^{\prime}$. Otherwise, transition $\left(s, a, s^{\prime}\right)$ does not cross $S^{\prime}$.

Definition 3. Let TS $=\left(S, E, A, s_{i n}\right)$ be a transition system. Let $S^{\prime} \subseteq S$ be a subset of states and $e \in E$ be an event. The following conditions (in the form of predicates) are defined for $S^{\prime}$ and $e$ :

$$
\begin{aligned}
\operatorname{in}\left(e, S^{\prime}\right) & \equiv \exists\left(s, e, s^{\prime}\right) \in A: s, s^{\prime} \in S^{\prime} \\
\operatorname{out}\left(e, S^{\prime}\right) & \equiv \exists\left(s, e, s^{\prime}\right) \in A: s, s^{\prime} \notin S^{\prime} \\
\operatorname{nocross}\left(e, S^{\prime}\right) & \equiv \exists\left(s_{1}, e, s_{2}\right) \in A: s_{1} \in S^{\prime} \Leftrightarrow s_{2} \in S^{\prime} \\
\operatorname{enter}\left(e, S^{\prime}\right) & \equiv \exists\left(s_{1}, e, s_{2}\right) \in A: s_{1} \notin S^{\prime} \wedge s_{2} \in S^{\prime} \\
\operatorname{exit}\left(e, S^{\prime}\right) & \equiv \exists\left(s_{1}, e, s_{2}\right) \in A: s_{1} \in S^{\prime} \wedge s_{2} \notin S^{\prime}
\end{aligned}
$$

Note that nocross $\left(e, S^{\prime}\right)=\operatorname{in}\left(e, S^{\prime}\right) \vee \operatorname{out}\left(e, S^{\prime}\right)$. We will abuse the notation and will use nocross $\left(e, S^{\prime},\left(s_{1}, e, s_{2}\right)\right)$ to denote a transition $\left(s_{1}, e, s_{2}\right)$ that makes the predicate nocross $\left(e, S^{\prime}\right)$ to hold, and the same for the rest of predicates.

The notion of a region is central for the synthesis of Petri nets. Intuitively, each region is a set of states that corresponds to a place in the synthesized Petri net, so that every state in the region models the marking of the place.

Definition 4 (Region). A set of states $r \subseteq S$ in transition system TS = $\left(S, E, A, s_{i n}\right)$ is called a region if the following two conditions are satisfied for each event $e \in E$ :

- (i) enter $(e, r) \Rightarrow \neg \operatorname{nocross}(e, r) \wedge \neg \operatorname{exit}(e, r)$

- (ii) $\operatorname{exit}(e, r) \Rightarrow \neg \operatorname{nocross}(e, r) \wedge \neg \operatorname{enter}(e, r)$

A region is a subset of states in which all transitions labeled with the same event $e$ have exactly the same "entry/exit" relation. This relation will become the successor/predecessor relation in the Petri net. The event may always be either an enter event for the region (case (i) in the previous definition), or always be an exit event (case (ii)), or never "cross" the region's boundaries, i.e. each transition labeled with $e$ is internal or external to the region, where 


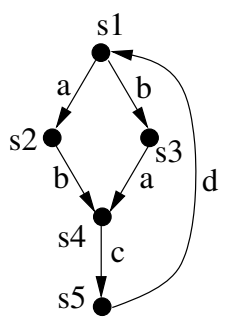

(a)

$$
\begin{aligned}
\text { Minimal Regions } & \\
\mathrm{r}_{1} & =\{\mathrm{s} 1, \mathrm{~s} 2\} \\
\mathrm{r}_{2} & =\{\mathrm{s} 1, \mathrm{~s} 3\} \\
\mathrm{r}_{3} & =\{\mathrm{s} 2, \mathrm{~s} 4\} \\
\mathrm{r}_{4} & =\{\mathrm{s} 3, \mathrm{~s} 4\} \\
\mathrm{r}_{5} & =\{\mathrm{s} 5\}
\end{aligned}
$$

(b)

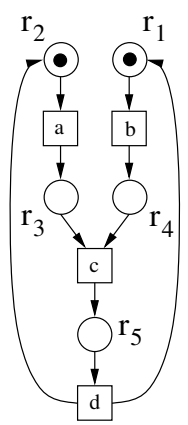

(c)

Fig. 1. (a) transition system, (b) minimal regions, (c) synthesis applying Algorithm of Figure 3.

the antecedents of neither (i) nor (ii) hold. The transition corresponding to the event will be successor, predecessor or unrelated with the corresponding place respectively. Examples of regions are reported in Figure 1: from the transition system of Figure 1(a), some regions are enumerated in Figure 1(b). For instance, for region $r_{2}$, event $a$ is an exit event, event $d$ is an entry event while the rest of events do not cross the region. Let $r$ and $r^{\prime}$ be regions of a transition system. A region $r^{\prime}$ is said to be a subregion of $r$ if $r^{\prime} \subset r$. A region $r$ is a minimal region if there is no other region $r^{\prime}$ which is a subregion of $r$. Going back to the example of Figure 1, in Figure 1(b) we report the set of minimal regions. Each transition system TS $=\left(S, E, A, s_{i n}\right)$ has two trivial regions: the set of all states, $S$, and the empty set. The set of non-trivial regions of TS will be denoted by $R_{\text {TS }}$.

A region $r$ is a pre-region of event $e$ if there is a transition labeled with $e$ which exits $r$. A region $r$ is a post-region of event $e$ if there is a transition labeled with $e$ which enters $r$. The sets of all pre-regions and post-regions of $e$ are denoted with ${ }^{\circ} e$ and $e^{\circ}$, respectively. By definition it follows that if $r \in{ }^{\circ} e$, then all transitions labeled with $e$ exit $r$. Similarly, if $r \in e^{\circ}$, then all transitions labeled with $e$ enter $r$.

The computation of the minimal regions is crucial for the synthesis methods in $[1,3]$. It is based on the notion of excitation region [6].

Definition 5 (Excitation region). The excitation region of an event e, $\operatorname{ER}(e)$, is the set of states in which e is enabled, i.e.

$$
\operatorname{ER}(e)=\left\{s \mid \exists s^{\prime}:\left(s, e, s^{\prime}\right) \in A\right\}
$$

In Fig. $1(\mathrm{a})$, the set $\operatorname{ER}(c)=\{s 4\}$ is an example of an excitation region ${ }^{1}$. The set of minimal regions sufficient for synthesis can be generated from the ERs of the events in a transition system in the following way: starting from the ER of

\footnotetext{
${ }^{1}$ Excitation regions are not regions in the terms of Definition 4 . The term is used for historical reasons. For instance, $\operatorname{ER}(c)$ is not a region.
} 

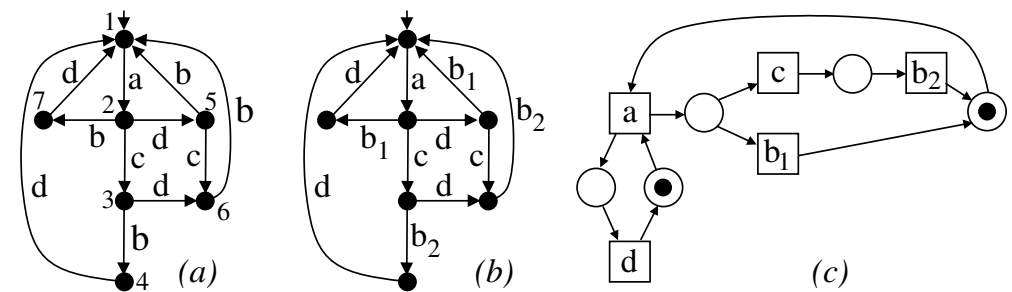

Fig. 2. (a) transition system (only numbers of states are shown), (b) ECTS by labelsplitting, (c) synthesized Petri net.

each event, set expansion is performed on those events that violate the region condition (a pseudocode of the expansion algorithm is given in Figure 10 in [3]). The following lemma characterizes the states to be added in the expansion of ERs:

Lemma 1 (Essential states to become a region [3]). Let TS = $\left(S, E, A, s_{i n}\right)$ be a transition system. Let $r \subset S$ be a set of states such that $r$ is not a region. Let $r^{\prime} \subseteq S$ be a region such that $r \subset r^{\prime}$. Let $e \in E$ be an event that violates some of the conditions for $r$ to be a region. The following predicates hold:

1. $\operatorname{in}(e, r) \wedge[\operatorname{enter}(e, r) \vee \operatorname{exit}(e, r)] \Longrightarrow$

$$
\left\{s \mid \exists s^{\prime} \in r:\left(s, e, s^{\prime}\right) \in A \vee\left(s^{\prime}, e, s\right) \in A\right\} \subseteq r^{\prime}
$$

2. enter $(e, r) \wedge \operatorname{exit}(e, r) \Longrightarrow$

$$
\left\{s \mid \exists s^{\prime} \in r:\left(s, e, s^{\prime}\right) \in A \vee\left(s^{\prime}, e, s\right) \in A\right\} \subseteq r^{\prime}
$$

3. out $(e, r) \wedge$ enter $(e, r) \Longrightarrow$

$$
\left[\left\{s \mid \exists s^{\prime} \in r:\left(s, e, s^{\prime}\right) \in A\right\} \subseteq r^{\prime}\right] \vee\left[\left\{s \mid \exists s^{\prime} \notin r:\left(s^{\prime}, e, s\right) \in A\right\} \subseteq r^{\prime}\right]
$$

4. $\operatorname{out}(e, r) \wedge \operatorname{exit}(e, r) \Longrightarrow$

$$
\left[\left\{s \mid \exists s^{\prime} \in r:\left(s^{\prime}, e, s\right) \in A\right\} \subseteq r^{\prime}\right] \vee\left[\left\{s \mid \exists s^{\prime} \notin r:\left(s, e, s^{\prime}\right) \in A\right\} \subseteq r^{\prime}\right]
$$

Hence, in cases 1 and 2 above, the violating event $e$ is converted into a nocross event, where only one way of expanding $r$ is possible. On the contrary, in case 3 (4) there are two possibilities for expansion, depending on whether the violating event will be converted into an enter or nocross (exit or nocross) event. In summary, set expansion to legalize violating events in a set of states generates a binary exploration tree. An example of such tree can be found in [3], Fig.12(b). The following definition formalizes the notion of essential set:

Definition 6 (Essential set of an event). Let TS $=\left(S, E, A, s_{i n}\right)$ be a transition system, and event $e \in E$. Essential(e) is the set of sets of states found by inductive application of Lem. 1, with initial set $\mathrm{ER}(e)$.

Notice that Essential(e) $\subseteq \mathcal{P}(S)$, and $\forall S_{i} \in \operatorname{Essential}(e)$ : $\operatorname{ER}(e) \subseteq S_{i}$. For instance, in Fig. 2(a) Essential $(c)=\{\operatorname{ER}(c)=$ $\{s 2, s 5\},\{s 1, s 2, s 5, s 7\},\{s 2, s 3, s 5, s 6\}\}$. The two states different from $\operatorname{ER}(c)$ in Essential(c) are computed according to case 4 in Lem. 1 on event $b$. 


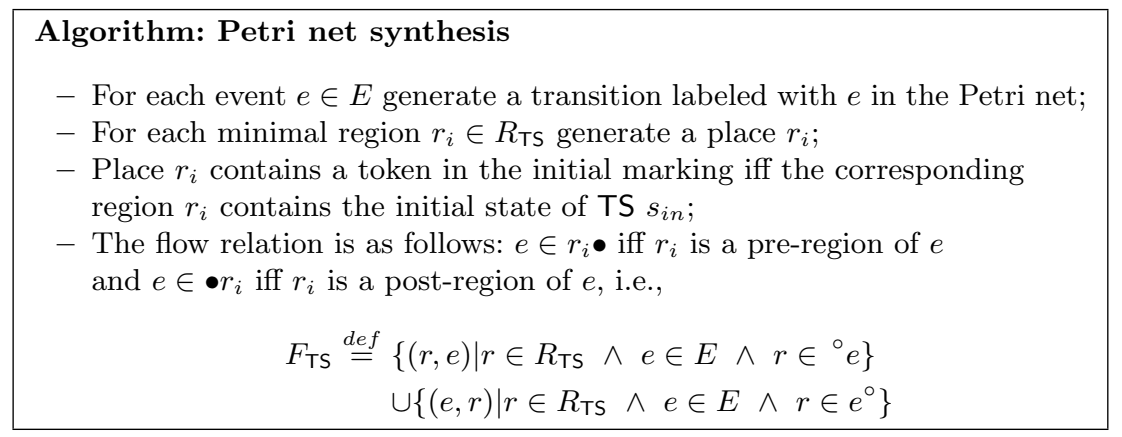

Fig. 3. Algorithm for Petri net synthesis from [7].

The procedure given by [7] to synthesize a Petri net, $N_{\mathrm{TS}}=$ $\left(R_{\mathrm{TS}}, E, F_{\mathrm{TS}}, R_{s_{i n}}\right)$, from an elementary transition system ${ }^{2}, \mathrm{TS}=\left(S, E, A, s_{i n}\right)$, is illustrated in Figure 3. Notice that only minimal regions are required in the algorithm [4]. An example of the application of the algorithm is shown in Figure 1. The initial transition system and the set of minimal regions is reported in Figures 1(a) and (b), respectively. The synthesized Petri net is shown in Figure $1(\mathrm{c})$.

\section{$2.3 \quad$ Excitation-closed transition systems}

Definition 7 (Excitation-closed transition systems). A transition system $\mathrm{TS}=\left(S, E, A, s_{i n}\right)$ is excitation-closed (ECTS) if it satisfies the following two axioms:

- Excitation closure (EC): For each event $a: \bigcap_{r \in{ }^{\circ} a} r=\operatorname{ER}(a)$

- Event effectiveness: For each event $a:{ }^{\circ} a \neq \emptyset$

The synthesis algorithm in Figure 3 applied to an ECTS derives a Petri net with reachability graph bisimilar to the initial transition system [3]. When the transition system is not excitation closed, then it must be transformed to enforce that property. One possible strategy is to represent every event by multiple transitions with the same label. This technique is called label splitting. Figure 2 illustrates the technique. The initial transition system, shown in Figure 2(a), is not an ECTS: the event $c$ is not EC (the only minimal region that contains $\operatorname{ER}(c)$ is $\{s 2, s 3, s 5, s 6\}$, which does not makes the EC axiom of Def. 7 to hold). The transition system is transformed by splitting the event $b$ into the events $b_{1}$ and $b_{2}$, as shown in Figure 2(b), resulting in an ECTS. The synthesized Petri net, with two transitions for event $b$ is shown in Figure 2(c). The reachability

${ }^{2}$ Elementary transition systems are a proper subclass of the transition systems considered in this paper, were additional conditions to the ones presented in Section 2.1 are required. 


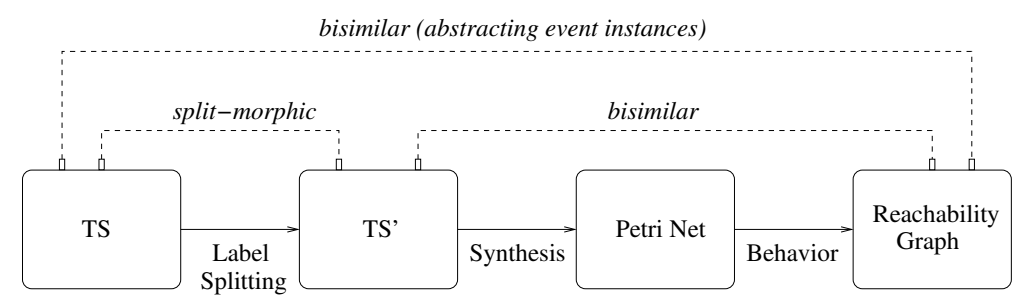

Fig. 4. Relationship between the different objects if label splitting is applied.

graph of the Petri net of Fig. 2(c) is split-morphic [3] to the transition system of Fig. 2(a): there exist a surjective mapping between the sets of events, where different instances of the same event $\left(a_{1}, a_{2}, \ldots\right)$ are mapped to the only one event $a$. If we abstract away the label indexes, the equivalence relation defined is bisimilarity. Fig. 4 shows the relationships between the original transition system, the transformed one obtained through label splitting, and the reachability graph of the synthesized Petri net.

Hence in Petri net synthesis label splitting might be crucial for the existence of a Petri net with bisimilar behavior. The following definition describes the general application of label splitting:

Definition 8 (Label splitting). Let TS $=\left(S, E, A, s_{i n}\right)$ be a transition system. The splitting of event $e \in E$ derives a transition system $\mathrm{TS}^{\prime}=\left(S, E^{\prime}, A^{\prime}, s_{i n}\right)$, with $E^{\prime}=E-\{e\} \cup\left\{e_{1}, \ldots, e_{n}\right\}$, and such that every transition $\left(s_{1}, e, s_{2}\right) \in A$ corresponds to exactly one transition $\left(s_{1}, e_{i}, s_{2}\right)$, and the rest of transitions for events different from $e$ in $A$ are preserved in $A^{\prime}$.

Label splitting is a powerful transformation which always guarantees excitation closure: any TS can be converted into one where every transition has a different label. By definition, the obtained TS is ECTS but the size of the derived Petri net is equal to the size of the obtained ECTS. In this paper we aim at reducing the number of splittings, thus reducing the size of the Petri net derived.

The work presented in this paper considers a particular application of the label splitting technique which is based on converting a set into a region, described in the next section. By restricting the transformation, we are able to determine these situations where a minimal set of labels is enough to guarantee excitation closure.

\section{Optimal label splitting to attain a region}

In this section the following problem is addressed: given a transition system $\mathrm{TS}=\left(S, E, A, s_{i n}\right)$ and a set of states $S^{\prime} \subseteq S$ which is not a region, determine the minimal number of label splittings to be applied in order to $S^{\prime}$ become a region. This is a crucial step for the technique presented in the following section to satisfy the ECTS condition. The main contribution of this section is 


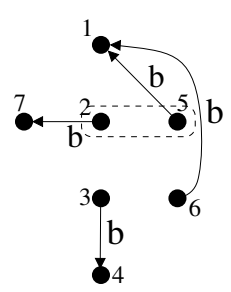

(a)

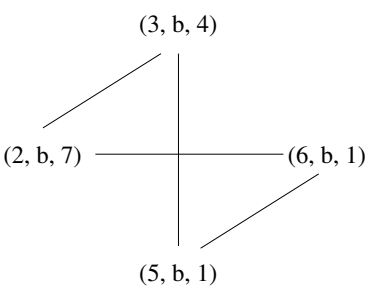

(b)

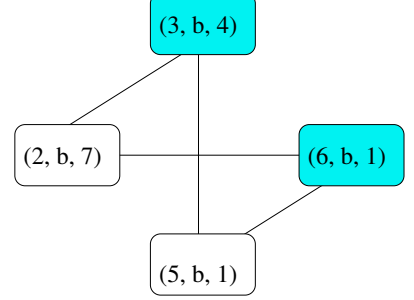

(c)

Fig. 5. (a) Projection of the transition system of Fig. 2(a) for the transitions on event $b$ and set of states $\left\{s_{2}, s_{5}\right\}$, (b) $\mathcal{G G}\left(b,\left\{s_{2}, s_{5}\right\}\right)$, (c) coloring.

to show that the problem might be reduced to compute the chromatic number of a graph [9].

First we introduce the concept of gradient graph:

Definition 9 (Gradient Graph). Given a transition system TS = $\left(S, E, A, s_{i n}\right)$, a set $S^{\prime} \subseteq S$ and an event $e \in E$, the gradient graph of $e$ with respect to $S^{\prime}$ in TS, denoted as $\mathcal{G G}\left(e, S^{\prime}\right)=\left(A_{e}, M\right)$ is an undirected graph defined as:

- $A_{e}=\left\{\left(s, x, s^{\prime}\right) \mid\left(s, x, s^{\prime}\right) \in A \wedge x=e\right\}$, is the set of nodes

- $M=\left\{\left(v, v^{\prime}\right) \mid v, v^{\prime} \in A_{e} \wedge\right.$

$\left[\left(\operatorname{enter}\left(e, S^{\prime}, v\right) \wedge\left(\operatorname{nocross}\left(e, S^{\prime}, v^{\prime}\right) \vee \operatorname{exit}\left(e, S^{\prime}, v^{\prime}\right)\right)\right) \vee\right.$

$\left.\left.\left(\operatorname{exit}\left(e, S^{\prime}, v\right) \wedge\left(\operatorname{nocross}\left(e, S^{\prime}, v^{\prime}\right) \vee \operatorname{enter}\left(e, S^{\prime}, v^{\prime}\right)\right)\right)\right]\right\}$, is the set of edges.

Informally, the gradient graph contains as nodes the transitions of an event $e$, and an edge exist between two nodes if they satisfy different predicates on set $S^{\prime}$. For instance, the gradient graph on event $b$ and set of states $S^{\prime}=\left\{s_{2}, s_{5}\right\}$ in the transition system of Fig. 2(a) is shown in Fig. 5(b) (for the sake of clarity we show in Fig. 5(a) the transitions on event $b$ from Fig. 2(a)).

A graph $G=(V, E)$ is $k$-colourable if there exists an assignment $\alpha: V \rightarrow\{1,2, \ldots k\}$ for which any pair of nodes $v, v^{\prime} \in V$ such that $\left(v, v^{\prime}\right) \in E$ satisfy $\alpha(v) \neq \alpha\left(v^{\prime}\right)$. The chromatic number, $\chi(G)$, of a graph $G$ is the minimum $k$ for which $G$ is $k$-colourable [9]. The rest of the section shows the relation between the chromatic number and the optimal label splitting to attain a region.

Definition 10 (Label splitting as gradient graph coloring). Given a transition system TS $=\left(S, E, A, s_{i n}\right)$, the label splitting of event e according to a coloring $\alpha$ of the gradient graph $\mathcal{G G}\left(e, S^{\prime}\right)$ derives the transition system $\mathrm{TS}^{\prime}=\left(S, E^{\prime}, A^{\prime}, s_{i n}\right)$ where $E^{\prime}=E-\{e\} \cup\left\{e_{1}, \ldots, e_{n}\right\}$, with $\left\{e_{1}, \ldots, e_{n}\right\}$ being the colors defined by the coloring of $\mathcal{G} \mathcal{G}\left(e, S^{\prime}\right)$. Every transition $\left(s, e, s^{\prime}\right)$ of event $e$ is transformed into $\left(s, e_{\alpha\left(\left(s, e, s^{\prime}\right)\right)}, s^{\prime}\right)$, whilst the rest of transitions of events in $E-\{e\}$ are preserved in $A^{\prime}$.

For instance, the label splitting of the transition system of Fig. 2(a) according to the coloring shown in Fig. $5(\mathrm{c})$ of $\mathcal{G} \mathcal{G}\left(b,\left\{s_{2}, s_{5}\right\}\right)$ is shown on Fig. 2(b). 
Proposition 1. Given a transition system $\mathrm{TS}=\left(S, E, A, s_{i n}\right)$ and the gradient graph $\mathcal{G G}\left(e, S^{\prime}\right)$. If event $e$ is split in accordance with a $\chi\left(\mathcal{G G}\left(e, S^{\prime}\right)\right)$-coloring of $\mathcal{G G}\left(e, S^{\prime}\right)$ then the new events $\left\{e_{1}, \ldots, e_{\chi}\left(\mathcal{G G}\left(e, S^{\prime}\right)\right)\right\}$ inserted satisfy the region condition on $S^{\prime}$ (c.f., Def. 4)).

Proof. By contradiction: assume there exists $e_{i} \in\left\{e_{1}, \ldots, e_{\chi}\left(\mathcal{G G}\left(e, S^{\prime}\right)\right)\right\}$ such that conditions of Def. 4 do not hold. Without loss of generality, we assume that there exist $\left(s_{1}, e_{i}, s_{2}\right)$ and $\left(s_{1}^{\prime}, e_{i}, s_{2}^{\prime}\right)$ for which predicates enter $\left(e_{i}, S^{\prime},\left(s_{1}, e_{i}, s_{2}\right)\right)$ and $\operatorname{nocross}\left(e_{i}, S^{\prime},\left(s_{1}, e_{i}, s_{2}\right)\right)$ hold (the other cases can be proven similarly). But then the nodes $\left(s_{1}, e_{i}, s_{2}\right)$ and $\left(s_{1}^{\prime}, e_{i}, s_{2}^{\prime}\right)$ are connected by an edge, but they are assigned the same color $e_{i}$. Contradiction.

Corollary 1. Given a transition system $\mathrm{TS}=\left(S, E, A, s_{\text {in }}\right)$ and a set $S^{\prime} \subseteq S$. If every event $e$ is split according to the colors required for achieving $\chi\left(\mathcal{G G}\left(e, S^{\prime}\right)\right)$, then $S^{\prime}$ is a region in the resulting transition system.

Proof. It follows from iterative application of Prop. 1.

In the following theorem we abuse the notation and extend the $\chi$ operator to sets of states:

Theorem 1. Given a transition system $\mathrm{TS}=\left(S, E, A, s_{i n}\right)$ and a set $S^{\prime} \subseteq S$, let $\chi\left(S^{\prime}\right)=\chi\left(\mathcal{G G}\left(e_{1}, S^{\prime}\right)\right)+\ldots+\chi\left(\mathcal{G G}\left(e_{n}, S^{\prime}\right)\right)$, with $E=\left\{e_{1}, \ldots, e_{n}\right\}$. Then $\chi\left(S^{\prime}\right)$ is a lower bound to the number of labels needed to make $S^{\prime}$ to be a region.

Proof. By contradiction: if there is a $k<\chi\left(S^{\prime}\right)$ such that only $k$ labels are needed to convert $S^{\prime}$ into region, then there is an event $e \in E$ for which less than $\chi\left(\mathcal{G G}\left(e, S^{\prime}\right)\right)$ labels are used to split $e$ for satisfying the conditions of Def. 4. This leads to a contradiction to the chromatic number of the graph $\mathcal{G G}\left(e, S^{\prime}\right)$.

The theory of this section represents the core idea for the label splitting technique of this paper. The next section shows how apply it to attain ECTSs.

\section{Optimal label splitting on essential sets for synthesis}

Given a non-ECTS, the following question arises: is there an algorithm to transform it into an ECTS with a minimal number of label splittings? This section addresses this problem, deriving sufficient conditions under which a positive answer can be given. As was done in previous work [3], in this paper we will restrict the theory to a particular application of the label splitting: instead of an arbitrary instantiation of Def. 8 which may split an event of a transition system in peculiar way, we will only consider the splittings used to convert essential sets into regions (Def. 10), technique which has been shown in the previous section.

We will tackle the problem in two phases: first, we will show how the EC for a given event can be achieved by using the essential sets found in the expansion of the ER of an event, defined in Lem. 1. Then we will show the conditions under which the strategy can be applied in the general case, i.e. considering the 
whole set of non-EC events. We start by defining the set of states that both are included in the intersection of pre-regions of an event but are not included in the excitation region:

$$
\text { Remainder }(e)=\left(\bigcap_{q \in{ }^{\circ} e} q\right) \backslash \operatorname{ER}(e)
$$

Clearly, event $e$ is EC if and only if Remainder $(e)=\emptyset$ (cf., Def. 7).

For the definitions and theorems below, we assume a context transition system TS $=\left(S, E, A, s_{i n}\right)$, and use Witness $(e)$ to denote the sets of essential sets of an event $e$ such that, if they are converted into regions, Remainder $(e)$ becomes empty. Formally:

Definition 11 (Witness set of an event). Let TS $=\left(S, E, A, s_{i n}\right)$ be a transition system, and event $e \in E$. Witness(e) is defined as follows:

$$
\begin{aligned}
\mathcal{C}=\left\{S_{1}, \ldots, S_{k}\right\} \in \text { Witness }(e) \Longleftrightarrow & \forall S_{i} \in \mathcal{C}: S_{i} \in \operatorname{Essential}(e) \wedge \\
& \left(\bigcap_{q \in\left({ }^{\circ} e \cup \mathcal{C}\right)} q\right) \backslash \operatorname{ER}(e)=\emptyset
\end{aligned}
$$

Finally, if $\mathcal{C}=\left\{S_{1}, \ldots, S_{k}\right\}$, we abuse the notation and use $\chi(\mathcal{C})$ to denote $\chi\left(\mathcal{G G}\left(e_{1}, S_{1}\right) \cup \ldots \cup \mathcal{G} \mathcal{G}\left(e_{1}, S_{k}\right)\right)+\ldots+\chi\left(\mathcal{G G}\left(e_{n}, S_{1}\right) \cup \ldots \cup \mathcal{G G}\left(e_{n}, S_{k}\right)\right)$, with $E=\left\{e_{1}, \ldots, e_{n}\right\}$. The union operator on gradient graphs is defined as $\mathcal{G G}\left(e, S_{1}\right) \cup$ $\ldots \cup \mathcal{G G}\left(e, S_{k}\right)=\left(A_{e}, M_{1} \cup \ldots \cup M_{k}\right)$, with $A_{e}$ and $M_{i}$ being the nodes and edges of the graph $\mathcal{G} \mathcal{G}\left(e, S_{i}\right)$, respectively c.f., Def. $9^{3}$.

First, we start by describing the minimal strategy to make an event to satisfy its excitation closure:

Proposition 2. Let $\mathcal{C}=\left\{S_{1}, \ldots, S_{k}\right\} \in W$ itness $(e)$ such that $\chi(\mathcal{C})$ is minimal, i.e., $\forall \mathcal{C}^{\prime} \in$ Witness $(e): \chi(\mathcal{C}) \leq \chi\left(\mathcal{C}^{\prime}\right)$. Then, if only label splitting on essential sets (Def. 10) is considered, $\chi(\mathcal{C})$ is the minimal number of labels needed to make Remainder $(e)=\emptyset$.

Proof. The minimality of $\chi(\mathcal{C})$ guarantees that, by using label splitting on essential sets, the minimal number of labels has been used in the coloring of the gradient graphs of each event for each set in $\mathcal{C}$.

Given a non-ECTS, the optimal label splitting problem on essential sets is to determine the sets to convert into regions in order to reduce the set Remainder to the empty set for each non-EC event, using minimal number of labels.

Definition 12 (Optimal label splitting on essential sets). Given the non-EC events $e_{1} \ldots e_{k} \in E$, define the universe $\mathcal{U}$ as Witness $\left(e_{1}\right) \quad \cup$ $\ldots \cup$ Witness $\left(e_{k}\right)$. The optimal label splitting problem is to determine sets $S_{1}, \ldots, S_{n} \subseteq \mathcal{U}$ such that $\forall 1 \leq i \leq k: \exists \mathcal{C} \in W$ itness $\left(e_{i}\right): \mathcal{C} \subseteq\left\{S_{1}, \ldots, S_{n}\right\}$ and where $\chi\left(S_{1}, \ldots, S_{n}\right)$ is minimal.

\footnotetext{
${ }^{3}$ We only consider the union of gradient graphs of the same event.
} 


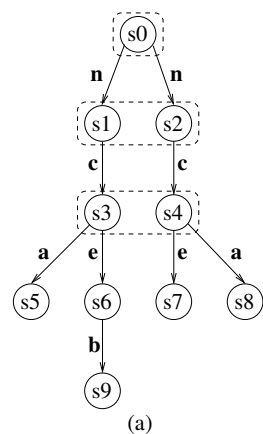

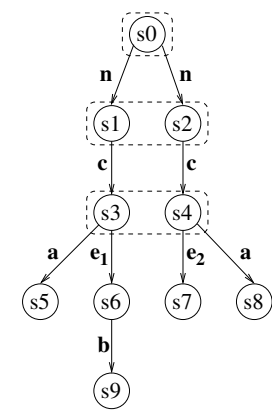

(b)

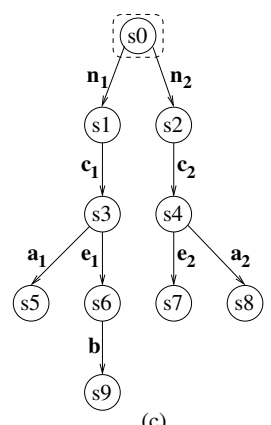

(c)

Fig. 6. (a) Initial transition system: all the events but $b$ are EC, (b) the splitting of $e$ leads to new events $e_{1}$ and $e_{2}$ not satisfying the EC property, (c) the final ECTS, where all events have been split.

Notice that Def. 12 is defined on the set of all essential sets of the nonEC events, searching for a set of essential sets which both has minimal number of labels and ensures EC for these events. An interesting result guarantees EC preservation for those events that both satisfy initially the EC and were not split:

Proposition 3. Let $e \in E$ be such that Remainder $(e)=\emptyset$ and it has not been selected for splitting. Then Remainder $(e)=\emptyset$ in the new transition system obtained after label splitting.

Proof. Label splitting preserves the set of regions: the predicates of Def. 4 that hold on each region will also hold if some event is split. Hence, the witness set of regions that ensures Remainder $(e)=\emptyset$ is still valid after label splitting.

However, label splitting may break events for which the excitation closure was satisfied or attained. Unfortunately, the new events appearing might not satisfy the excitation closure property as the following example demonstrates.

Example 1. In the transition system of Figure 6(a) events $n, c, a$ and $e$ are EC, as demonstrated by their witness: Witness $(n)=\{\{s 0\}\}$, Witness $(c)=\{\{s 1, s 2\}\}$, Witness $(a)=W i$ tness $(e)=\{\{s 3, s 4\}\}$. However, $b$ is not EC, and the splitting required for $b$ to be $\mathrm{EC}$ is done on the essential set $\{s 6\}$, which requires to split the $\mathrm{EC}$ event $e$, resulting in the transition system of Fig. 6(b). The new events $e_{1}$ and $e_{2}$ arising from the splitting of $e$ are not EC. This requires further splittings, which as in the case of $b$, force the splitting of EC events resulting in new events that are not EC. Four iterations are required to attain the ECTS shown in Fig. 6(c).

This example invalidates any label splitting strategy that aims at reaching excitation closure in just one iteration of the synthesis process: in general, when the splitting of some event is applied, its ER is divided into several ERs for which there might be no witness which guarantee the EC of these new events arising. 
Importantly, the label splitting technique preserves the regions, but new regions might be necessary for the new events arising from a splitting. Therefore, any label splitting technique must be an iterative method (see next section for such a method). However, if the new labels inserted do not incur excitation closure problems, the presented technique guarantees the optimal label splitting:

Theorem 2. Let $\mathrm{TS}^{\prime}=\left(S, E^{\prime}, A^{\prime}, s_{i n}\right)$ be the transition system reached after splitting labels on non-EC events $e_{1}, \ldots e_{k}$ in transition system TS = $\left(S, E, A, s_{i n}\right)$, using the strategy from Def. 12. Then, if the new events appearing are $\mathrm{EC}$, the number of splittings performed is minimal and $\mathrm{TS}^{\prime}$ is $\mathrm{ECTS}$.

Proof. The minimality of the strategy used in Def. 12 ensures that no less splittings are possible to make $e_{1}, \ldots e_{k}$ EC. Moreover, the assumption implies that the new events arising from the splitting are EC. Finally, Prop. 3 guarantees that EC events that were not split are still EC. The set of events $E^{\prime}$ is partitioned into these three sets, and therefore TS $^{\prime}$ is ECTS.

\section{$5 \quad$ A greedy algorithm for iterative label splitting}

The optimization problem for optimal label splitting presented in the previous section is hard to tackle: considering the global optimal label splitting through local achievement of EC for every non-EC event. However, if some relaxations are done, the problem can be seen as an instance of the weighted set cover (WSC) problem [2]. This section shows how to cast the problem into an WSC setting and depicts an algorithm for label splitting that iterates until excitation closure is attained. Unfortunately, for a set of witness elements $S_{1}, \ldots, S_{n}$, the equality

$$
\chi\left(S_{1}, \ldots, S_{n}\right)=\chi\left(S_{1}\right)+\ldots+\chi\left(S_{n}\right)
$$

does not hold in general, e.g., imagine that some $S_{i}, S_{j}$ satisfy $S_{i} \cap S_{j} \neq \emptyset$ : in that situation one may be counting twice the labels (colors) needed in the right part of the equality of (1). That prevents an exact mapping of the label splitting optimization problem into the WSC setting. However, one may allow this imprecision for the sake of having an efficient manner to derive good candidates. The following model maps the optimization problem of Def. 12 into an ILP problem, whose solution provides candidates that, although potentially suboptimal, might represent good candidates for label splitting in practice:

$$
\begin{gathered}
\min \sum_{\mathcal{C} \in \mathcal{W}} \chi(\mathcal{C}) \cdot X_{\mathcal{C}} \\
\text { s.t. } \\
\forall e \in e_{1} \ldots e_{k}: \sum_{\mathcal{C} \in W \text { itness }(e)} X_{\mathcal{C}} \geq 1 \\
X_{\mathcal{C}} \in\{0,1\}
\end{gathered}
$$

where $e_{1} \ldots e_{k} \in E$ are the set of non-EC events and $\mathcal{W}=$ Witness $\left(e_{1}\right) \cup \ldots \cup$ Witness $\left(e_{k}\right)$. A solution to the ILP model (2) will then minimize the sum of the cost of the witness selected for each non-EC event. 


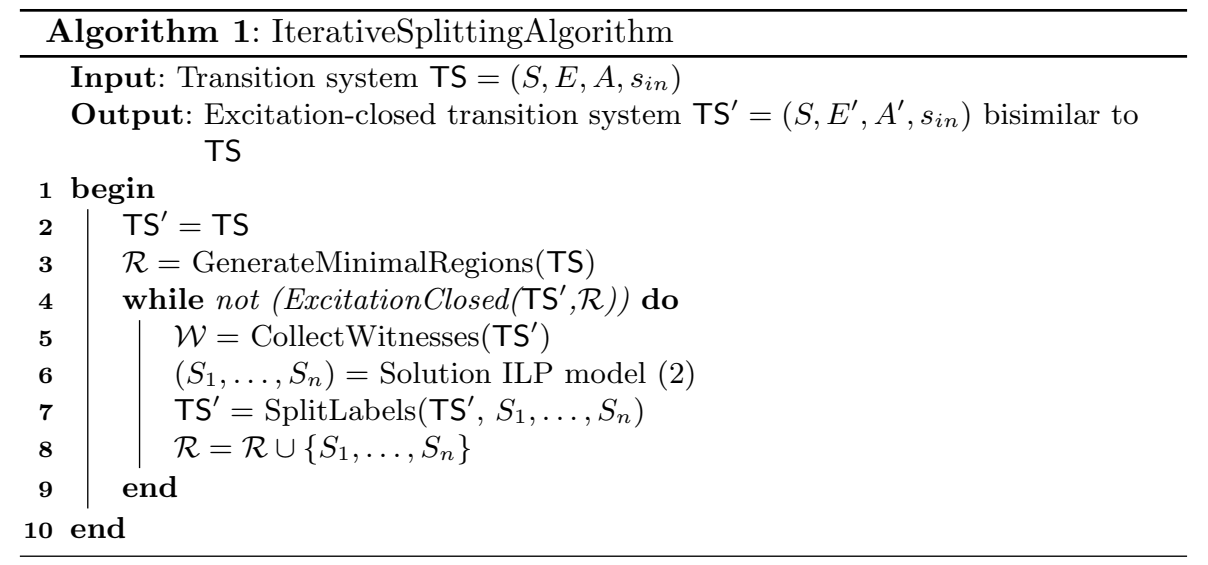

Algorithm 1 presents the iterative strategy to derive an ECTS. It first computes the minimal regions of the initial transition system. Then the main loop of the technique starts by collecting the witnesses for non-EC events of the current transition system, which are provided in the set $\mathcal{W}$ (line 5). Then the cover for the non-EC events is computed by solving the ILP model (2) in line 6. Notice that for the sake of clarity of the algorithm, we provide in line 6 the sets that form the cover instead of providing the particular witness selected for each event (i.e., given a solution $\mathcal{C}_{1}, \ldots, \mathcal{C}_{k}$ of model $\left.(2),\left\{S_{1}, \ldots, S_{n}\right\}=\bigcup_{1<i<k} \mathcal{C}_{i}\right)$. In line 7 the splitting of labels corresponding to $\chi\left(S_{1}, \ldots, S_{n}\right)$ is performed, ensuring that sets $S_{1}, \ldots, S_{n}$ become regions. The new regions are appended to the regions found so far (which are still regions, see proof of Prop. 3), and the excitation closure is re-evaluated to check convergence.

Although the presented iterative technique is not guaranteed to provide the optimal, the improvements with respect to the previous (also non-optimal) approaches $[1,3]$ are:

- the whole set of non-EC events are considered in every iteration: in previous work only one is considered, and

- for every non-EC event, the necessary splittings are applied to attain excitation closure: on the previous work, only one of the splittings was applied.

Hence the macro technique presented in this section is meant to speed-up the achievement of the excitation closure, when compared to the micro techniques presented in the literature.

\section{Discussion and conclusions}

This paper has presented a fresh look at the problem of label splitting, by relating it to some of the well-known NP-complete problems like chromatic number or 
set covering. In a restricted application of label splitting based on essential sets, optimality is guaranteed if certain conditions hold.

For the sake of clarity, we have restricted the theory to safe Petri nets. The extension to $k$-bounded Petri nets can be done by adapting the notion of gradient graph to use gradients (see [1] for the formal definition of gradient) instead of the predicates required in Def. 4 . Given a multiset $r$ which is not a $k$-bounded region, the essential sets will be those multisets $r^{\prime} \geq r$ such that the number of gradients for some event has decreased, i.e., $r^{\prime}$ is a sound step towards deriving a region from multiset $r$. Finally, the excitation closure definition for the general case should be the one described in [1], and the witness of an event defined accordingly.

As a future work, addressing the general problem with unrestricted application of splitting (i.e., not using essential sets but instead arbitrary selections of labels to split) might be an interesting direction to follow. Also, incorporating the presented techniques into our synthesis tool [1] will be considered.

\section{References}

1. J. Carmona, J. Cortadella, and M. Kishinevsky. New region-based algorithms for deriving bounded Petri nets. IEEE Transactions on Computers, 59(3):371-384, 2009.

2. T. H. Cormen, C. Stein, R. L. Rivest, and C. E. Leiserson. Introduction to Algorithms. McGraw-Hill Higher Education, 2001.

3. J. Cortadella, M. Kishinevsky, L. Lavagno, and A. Yakovlev. Deriving Petri nets from finite transition systems. IEEE Transactions on Computers, 47(8):859-882, Aug. 1998.

4. J. Desel and W. Reisig. The synthesis problem of Petri nets. Acta Inf., 33(4):297315, 1996.

5. A. Ehrenfeucht and G. Rozenberg. Partial (Set) 2-Structures. Part I, II. Acta Informatica, 27:315-368, 1990.

6. M. Kishinevsky, A. Kondratyev, A. Taubin, and V. Varshavsky. Concurrent Hardware: The Theory and Practice of Self-Timed Design. John Wiley and Sons, London, 1993.

7. M. Nielsen, G. Rozenberg, and P. Thiagarajan. Elementary transition systems. Theoretical Computer Science, 96:3-33, 1992.

8. C. A. Petri. Kommunikation mit Automaten. PhD thesis, Bonn, Institut für Instrumentelle Mathematik, 1962. (technical report Schriften des IIM Nr. 3).

9. D. B. West. Introduction to Graph Theory. Prentice-Hall, 1996. 